\title{
Qualitative Ansätze der Demokratiemessung
}

\author{
von Hans-Joachim Lauth
}

\begin{abstract}
Nicht nur quantitative Messungen der Demokratiequalität haben eine Bedeutung in der empirischen Demokratieforschung. Von zunehmender Relevanz sind auch qualitative oder komplexe Ansätze der Demokratiemessung, die in der Lage sind, ein vielschichtiges Qualitätsprofil von Demokratien zu bestimmen. Vorgestellt werden Forschungsansätze, die sich aus unterschiedlicher Perspektive dieser Aufgabe stellen. Neben dem konzeptionellen und methodischen Design werden auch empirische Befunde referiert. Der kritischen Analyse dieser Ansätze folgt die Diskussion über den möglichen Einbezug von Umfragedaten aus dem Bereich der politischen Kulturforschung in die Bewertung der Qualität von Demokratien. Abschließend werden bestehende Forschungsaufgaben skizziert.
\end{abstract}

While the importance of quantitative measures of democracy should not be underestimated, qualitative or complex approaches are of increasing relevance due to their capability in analysing democratic systems and societies on a number of different levels. This contribution presents several approaches that address this challenge from a variety of perspectives. In addition to a conceptual and methodological discussion, empirical results will be presented. Furthermore, the potential value of survey data for an analysis of the quality of democracy will be discussed, leading to the presentation of a timely research agenda.

\section{Einleitung}

Die begrenzten Aussagemöglichkeiten von quantifizierenden Demokratiemessungen verdeutlichen die Notwendigkeit, auch qualitative Ansätze in die Bestimmung der Qualität einer Demokratie einzubeziehen. ${ }^{1}$ Die Bezeichnung qualitativ wird oftmals gewählt, weil solche Ansätze auf eine quantifizierende Darstellung verzichten. Doch dies ist inzwischen nicht mehr die Regel. Der zentrale Unterschied besteht eher in der Komplexität der Messanlage. Während es im Bereich der sogenannten quantifizierenden Demokratiemessung möglich ist, große Fallzahlen zu bearbeiten, benötigen qualitativ gelagerte Messungen einen weitaus größeren Forschungsaufwand, um nur wenige Fälle bearbeiten zu kön-

1 Siehe dazu die Übersicht von Lauth, H.-J.: Möglichkeiten und Grenzen der Demokratiemessung, in: ZSE, 4 (2010), 498-529 und Müller, T./Pickel, S.: Wie lässt sich Demokratie am besten messen? Zur Konzeptqualität von Demokratie-Indizes, in: PVS, 3 (2007), 511-539. 
nen. Oftmals liegen daher auch (nur) Einzelfallstudien vor. Kein prinzipieller Unterschied besteht in der Datenerhebung. Grundsätzlich ist darauf hinzuweisen, dass in beiden Forschungsperspektiven qualitative Methoden zum Einsatz kommen und die Bewertung grundsätzlich subjektiv erfolgt, also auf einer individuellen Bewertung (judgement) beruht, die sich allerdings aufgrund der Transparenz des Verfahrens zu einem gewissen Maße objektivieren lässt. Die Komplexität qualitativer Demokratiemessung betrifft zum einen die Differenziertheit der Messanlage selbst und zum anderen die Menge der zu erhebenden Daten.

Die Zahl der Messvorschläge ist im Bereich der qualitativen Demokratiemessung deutlich kleiner, was nicht zuletzt mit dem damit verbundenen Forschungsaufwand in Verbindung stehen dürfte. Der wirkungsmächtigste Ansatz ist ohne Zweifel der Democratic Audit. Er geht auf Überlegungen zurück, die Qualität der britischen Demokratie zu bestimmen, um Defizite identifizieren und soweit möglich beheben zu können. ${ }^{2}$ Eine Reihe von Studien ist diesem Unternehmen entsprungen, das von David Beetham, Kenneth Boyle und Stuart Weir sowie weiteren Mitarbeitern getragen wurde. Ihren Niederschlag gefunden haben die Forschungsbemühungen auch im Rahmen der UN und in einem komparativen Forschungsprojekt, das zunächst acht (überwiegend außereuropäische) Länder umfasst und von der in Stockholm ansässigen Einrichtung „IDEA“ (Institute for Democracy and Electoral Assistance) getragen wird. ${ }^{3}$ Inzwischen wurde auch das IDEA-Projekt weiterentwickelt und bedarf einer separaten Betrachtung. Neben diesen Forschungen werden zwei Ansätze aus dem deutschsprachigen Raum aufgegriffen: zum einen der Messvorschlag zu einem kontextualisierten Maß für Demokratie, der zunächst von Heidrun Abromeit entwickelt und dann von Michael Stoiber aufgegriffen und weiter bearbeitet wurde, ${ }^{4}$ und zum anderen die vom Autor entworfene Demokratiematrix, die Qualitätsprofile demokratischer Systeme zu bestimmen sucht. ${ }^{5}$ Alle genannten Ansätze reflektieren weitaus stärker als im Bereich der quantifizierenden Messungen die Kontextrelevanz des Vorgehens, wobei sie aber zu unterschiedlichen Lösungsvorschlägen kommen.

2 Vgl. http://www.democraticaudit.com/index.php (20.02.2011).

3 Beetham, D./Boyle, K.: Introducing Democracy. 80 Questions and Answers, Cambridge, 1995; Beetham, D./Bracking, S./Kearton, I./Weir, S.: International IDEA Handbook on Democracy Assessment, The Hague, 2002.

4 Abromeit, H.: Die Messbarkeit von Demokratie. Zur Relevanz des Kontexts, in: PVS, 1 (2004), 73-93; Stoiber, M.: Ein neues, kontextualisiertes Maß für Demokratie. Konzeptualisierung und Operationalisierung, in: ZPol, 18/2 (2008), 209-231.

5 Lauth, H.-J.: Demokratie und Demokratiemessung. Eine konzeptionelle Grundlegung für den interkulturellen Vergleich, Wiesbaden, 2004. 
Nicht vorgestellt werden Messkonzeptionen, die verstärkt die outputKomponente (Performanz) eines politischen Systems zur Bestimmung der Qualität einer Demokratie heranziehen, da diese nicht mehr das prozeduralistische Grundverständnis von Demokratie teilen. ${ }^{6}$ Hinzuweisen bleibt schließlich auf ein umfangreiches Forschungsprojekt (mit Ausrichtung auf USAID), dessen Grundkonzeption bislang nur skizziert wurde. ${ }^{7}$

\section{Qualitative Ansätze}

\section{Democratic Audit}

Der Democratic Audit basiert auf einem Forschungsverbund, der am Human Rights Centre der Universität von Essex angesiedelt ist. Dort findet sich auch ein guter Überblick über die Forschungstätigkeit. ${ }^{8}$ Eine Besonderheit dieses AuditProjekts besteht in seinem politischen Anspruch, der auch auf politische Bildung im weiteren Sinne abzielt. Durch die Beteiligung von Bürgern und Basisgruppen an der Erhebung und Auswertung der empirischen Befunde sollen Teile der Zivilgesellschaft zu einem reflexiven Umgang mit ,ihrer“ Demokratie veranlasst werden, der sowohl ihr Wissen als auch ihre Bereitschaft zu politischem Engagement mehren soll. Ziel ist die vertiefte Demokratisierung des jeweiligen Landes.

Konzeptioneller Ausgangspunkt des Forschungsprojektes ist die Bestimmung der Prinzipien und Indikatoren der Demokratie. ${ }^{9}$ Demokratie wird mittels zweier Dimensionen bestimmt: popular control (oder staatsbürgerliche Souveränität) und politische Gleichheit. Kontrolle wird verstanden als das Recht, über politi-

6 Campbell, D.F.J./Barth, T.D.: Wie können Demokratie und Demokratiequalität gemessen werden? Modelle, Demokratie-Indices und Länderbeispiele im globalen Vergleich, in: SWS-Rundschau, 49/2 (2009), 209-233; Konrad-Adenauer-Stiftung (Hg.): Índice de Desarollo Democrático de América Latina, IDD-Lat 2005, Buenos Aires, 2005. Auch der Report UNDP: Democracy in Latin America. Towards a Citizens' Democracy, New York/Buenos Aires, 2004, schließt umfangreich die soziale und wirtschaftliche Dimension ein. Zugleich liefert er eine Menge an relevanten Befunden zu demokratischen Entwicklungen, die jedoch nicht zu einem übergreifenden Demokratieindex zusammengefasst werden. Erstellt werden hingegen ein Index of Electoral Democracy und ein Democratic Support Index.

7 Gerring, J./Coppedge, M.: Measuring Democracy: A Multidimensional, Tiered, and Historical Approach, Conference Paper, 2009.

8 Vgl. unter www.democraticaudit.com.

9 Beetham, D.: Key Principles and Indices for a Democratic Audit, in: Beetham, D. (Hg.): Defining and Measuring Democracy, London, 1994, 25-43; Klug, F./Starmer, K./Weir, S.: The Three Pillars of Liberty. Political Rights and Freedoms in the United Kingdom, London, 1996; Beetham, D./Weir, S.: Political Power and Democratic Control in Britain, London, 1999. 
sche Inhalte (Gesetze) oder über die Auswahl von Repräsentanten zu entscheiden und diese zu kontrollieren. Die politische Gleichheit erschließt sich durch gleiches aktives und passives Wahlrecht und durch die Chancengleichheit in der Artikulation aller Präferenzen, die sich zugleich auf ihre politische Wahrnehmung und Behandlung in der Gesetzgebung bezieht. Beide zentrale Dimensionen oder demokratische Prinzipien werden durch vier Unterdimensionen präzisiert: ${ }^{10}$

- Wahlen oder ,free and fair elections“

- Regierungsinstitutionen oder „open and accountable government“

- Bürgerrechte oder ,civil and political rights“

- Gesellschaftliche Demokratie oder ,democratic society“

Diese Unterdimensionen werden mit 30 Fragen oder Indikatoren weiter konkretisiert, die sowohl formale Regeln als auch die gegenwärtige Praxis berücksichtigen. In der Untersuchung von „freien und fairen Wahlen“ werden in verschiedenen Aspekten ihre Inklusivität und die Effektivität der Regierungsmacht erfasst. Accountability bezieht sich auf drei Ebenen: auf die politische (Rechtfertigung und Verantwortlichkeit der Regierung vor dem Parlament), die legale (rechtsstaatliches Handeln der staatlichen Akteure) und die finanzspezifische (Finanzkontrolle über Regierung durch Parlament und Gerichtsbarkeit). Grundlage einer effektiven accountability sind Gewaltenteilung und Transparenz des Regierungshandelns. Die dritte (Unter-)Dimension (,bürgerliche und politische Rechte") betrifft die klassischen Freiheitsrechte. Die „demokratische Gesellschaft" beinhaltet nicht nur die Sphäre der Zivilgesellschaft, sondern bezieht die Repräsentativität der Medien und die demokratische Struktur von mächtigen Privatunternehmen sowie den demokratischen Charakter der politischen Kultur und des Erziehungssystems mit ein.

Als Indikatoren finden quantitative Variablen (Wahlbeteiligung und Befunde zur politischen Kultur) zwar Eingang in die Untersuchung, insgesamt werden jedoch hauptsächlich qualitative, auf subjektiven Einschätzungen beruhende items verwendet. Abgelehnt wird eine Übertragung der Ergebnisse auf eine gemeinsame quantitative Skala. Entsprechend findet sich keine Berechnung eines Aggregatoder Indexwertes. Verzichtet wird gleichfalls auf eine Skalierung der einzelnen Fragen. Damit erhöht sich allerdings die Schwierigkeit, angemessen mit den Fragen zu arbeiten, um das Maß einer Demokratie zu bestimmen. Um die Problematik der Reliabilität zu reduzieren, präzisieren die Autoren den Maßstab für 
eine „gute“ Demokratie. Herangezogen werden dazu erstens die international (oder zumindest regional) akzeptierten Menschenrechtsstandards, wie sie in den verschieden Chartas und Konventionen niedergelegt wurden. ${ }^{11}$ Zweitens lässt sich ein Maßstab aus der Beobachtung der best practice in anderen Ländern gewinnen (dies gilt etwa für die Transparenz und Offenheit des staatlichen Handelns im Fall der USA und Schwedens). Damit soll gewährleistet sein, dass nicht utopische, sondern realistische und realisierbare Kriterien verwendet werden. Eine rein theoretisch konstruierte Messskala (oder ideal standard) wird abgelehnt, da sie zu wenig Bezug zur empirischen Wirklichkeit und Brauchbarkeit habe. $^{12}$

Insgesamt kann der Versuch, den Maßstab der Demokratie auf den verschiedenen Ebenen (der Prinzipien, Dimensionen und Indikatoren) so weit zu präzisieren, dass er für eine empirische Anwendung brauchbar ist, nicht ganz befriedigen. ${ }^{13}$ Beide Grundprinzipien sind sehr abstrakt formuliert, verschiedene Möglichkeiten der Konkretisierung bleiben ungenutzt. ${ }^{14}$ Beetham selbst weist auf diesen Sachverhalt hin: „The definition of democracy in terms of the two general principles of popular control and political equality does in theory allow for their institutionalization in different ways; and enables us to recognize democracy as an aspiration in many different societies and in various historical forms. "15 Viele Aspekte bleiben vage und werden durch die Fragestellung eher implizit angesprochen, ohne dass sie ausreichend konkretisiert würden. Während ein breites Verständnis von demokratischer Kontrolle vorliegt, ist der Begriff der Freiheit eher unterrepräsentiert. Zudem thematisiert der Democratic Audit nicht nur Facetten der Demokratiequalität, sondern auch der Konsolidierung einer Demokratie (Frage 30) oder Aspekte von demokratieförderlichen Faktoren (Frage 29).

11 Beetham, D., a.a.O., 26f., 32f.; Beetham, D./Weir, S.: Democratic Audit in Comparative Perspective, in: Lauth, H.-J./Pickel, G./Welzel, C. (Hg.): Demokratiemessung, Opladen, 2000, 73-88.

12 Beetham, D., a.a.O., 33.

13 Lauth, H.-J.: Demokratie und Demokratiemessung, a.a.O., 287ff. Es ist allerdings darauf hinzuweisen, dass sich mehr Hinweise in den konkreten Untersuchungen finden (Klug, F./Starmer, K./Weir, S., a.a.O.; Beetham, D./Weir, S., a.a.O.), in denen unter anderem international standards hinsichtlich der einzelnen Kriterien aufgegriffen werden.

14 Die eingeschlagene Operationalisierungsstrategie gibt Anlass, von einer ,institutionellen Lücke“ zu sprechen. Zu Recht weist Schedler darauf hin, dass die Verbindung zwischen der Formulierung der abstrakten Prinzipien und den konkreten Operationalisierungsfragen nicht hinreichend ausgearbeitet wurde (Schedler, A.: Dimensionen der Demokratiequalität. Keine abschließenden Bemerkungen, in: Campbell, D.F.J./Liebhart, K./Martinsen, K./Schaller, C./Schedler, A. (Hg.): Die Qualität der österreichischen Demokratie. Versuche einer Annäherung, Wien, 1996, 165-180, hier 169).

15 Beetham, D., a.a.O., 40. 
Problematisch ist die vierte Dimension (democratic society), da hier erkennbar der Bereich des rein Politischen verlassen wird. Repräsentativität der Medien, interne Demokratie von privaten Körperschaften, ein gehobenes politisches Bewusstsein der Bürger, das praktizierte Ausmaß an Partizipation, der demokratische Charakter der politischen Kultur und des Bildungssystems ${ }^{16}$ sind Faktoren, die einerseits meist nur in anspruchsvolleren normativen Demokratiekonzepten enthalten sind und andererseits in Teilen durchaus als umstritten gelten dürfen. Letzteres betrifft auch die Mitwirkung der unteren politischen Ebenen. Föderale oder dezentrale Strukturen werden eindeutig bevorzugt. Andere Bereiche - wie Medien und Kommunikationsfreiheiten oder rule of the law - werden in den 30 Fragen kaum explizit und wenig systematisch angeführt. Schließlich stellt sich bei einigen Indikatoren (wie bei dem Ausmaß der Wahlbeteiligung) die Frage der Validität.

Die entscheidende Schwäche besteht aber im Fehlen eines Maßstabes, der die Leitidee präzisieren könnte. Der Rückgriff auf international oder best standards, die in den empirischen Studien zum Tragen kommen, kann diese Lücke nur teilweise schließen und verschleiert zudem die bei konkurrierenden Vorschlägen notwendige Leitidee zur Begründung der ausgewählten Standards. Die Schwierigkeit, das empirische Konzept umzusetzen, lässt sich unter anderem daran ablesen, dass es mehrjähriger Forschungsanstrengungen und umfangreicher Publikationen bedurfte, um den Audit für ein einziges Land vollständig zu bearbeiten. Inzwischen sind auch kulturelle und soziale Menschenrechte in den Fokus der Untersuchung gerückt. ${ }^{17}$

Die beschriebene Messanlage hat nach Zählung der Verantwortlichen in bislang 18 Fallstudien Anwendung gefunden. Die Fragen von Beetham werden als wesentliche Grundlagen für den Democratic Audit in Großbritannien herangezogen, der inzwischen in drei umfangreichen Bänden zu Freiheitsrechten, zu politischer Macht und demokratischer Kontrolle sowie zur Demokratie unter Blair dokumentiert wurde. ${ }^{18}$ Abgesehen von der bleibenden Problematik des Maßstabes gelingt es, mit dem Frageraster ein differenziertes Bild der britischen Demokratie mit ihren Schwächen und Stärken zu zeichnen, das in seiner Bestandsaufnahme weit über die begrenzte Komplexität der anderen Ansätze der Demokratiemes-

16 Beetham, D., a.a.O., 30.

17 Weir, S.: Unequal Britain: Human Rights as a Route to Social Justice, London, 2006.

18 Klug, F./Starmer, K./Weir, S., a.a.O.; Beetham, D./Weir, S., a.a.O.; Beetham, D./Byrne, I./Ngan, P./Weir, S.: Democracy Under Blair. A Democratic Audit of the United Kingdom, London, 2002. 
sung hinausreicht und somit eine Unterscheidung innerhalb der Gruppe von Demokratien ermöglicht, die ansonsten oftmals in einer Kategorie zusammengefasst werden.

Der empirische Befund kann hier nicht umfassend gewürdigt werden, doch ist die grundlegende Einschätzung der britischen Demokratie knapp zu skizzieren. Als eine spezifische Schwachstelle wird die nicht befriedigende Verankerung von Menschenrechten gesehen, was ihre Kodifizierung, den Rechtsweg und ihre gleiche Geltung für alle gesellschaftlichen Gruppen betrifft. Bemängelt werden zugleich die begrenzte Transparenz des Regierungsapparates und die geringen institutionellen Kontrollmöglichkeiten. Das Urteil fällt hart aus: „In the absence of these [checks and balances], 'electoral democracy' degenerates all too readily into elective dictatorship, as we have witnesses once again under Tony Blair". ${ }^{19}$ Diese negative Beurteilung entspricht jedoch nicht dem Gesamtbefund der Untersuchung. Eine differenzierte Einschätzung ergibt die graphische Darstellung der Befunde, die sich an dem Fragenkatalog ausrichtet (vgl. Abb. 1). Demnach werden die Stärken der britischen Demokratie in der aktiven Ausprägung der Zivilgesellschaft gesehen. Deutlich positiv werden auch die zivile Kontrolle über die Sicherheitsorgane, die Bekämpfung der Korruption und die Rechtsstaatlichkeit eingeschätzt, während Transparenz und accountability der Regierung kontinuierlich skeptisch bewertet werden. Ähnliches gilt für den Devolutionsprozess, die Responsivität der Regierung und die Repräsentation von Frauen im öffentlichen Leben, wenngleich hier Fortschritte verzeichnet wurden. Generell wird trotz der harschen Kritik an der Regierung Blair ein Fortschritt in der demokratischen Entwicklung seit Thatcher konstatiert. In zwei Bereichen werden jedoch signifikante Gegentrends moniert: So sinken sowohl die Wahlbeteiligung als auch die demokratische Qualität der Parteiorganisationen.

Auch zu Schweden wurden auf Grundlage des Audit parallel zum britischen Projekt Untersuchungen zur Qualität der Demokratie vom Center for Business and Policy Studies (Stockholm - SNS) durchgeführt, die jedoch vom Umfang her geringer ausfallen. Dabei zeigt sich ein eher positives Bild der schwedischen Demokratie; die liberalen Freiheiten, die Rechtsstaatlichkeit, die lebendige Zivilgesellschaft, die hohe Repräsentativität der politischen Führung und die politische Entscheidungskapazität werden wohlwollend gekennzeichnet.

19 The Democratic Audit: Failing Democracy. Democratic Findings No. 7, Human Rights Centre: University of Essex, 2005, 1-4, hier 1. 
Abbildung 1: Qualität der britischen Demokratie nach dem Democratic Audit ${ }^{20}$

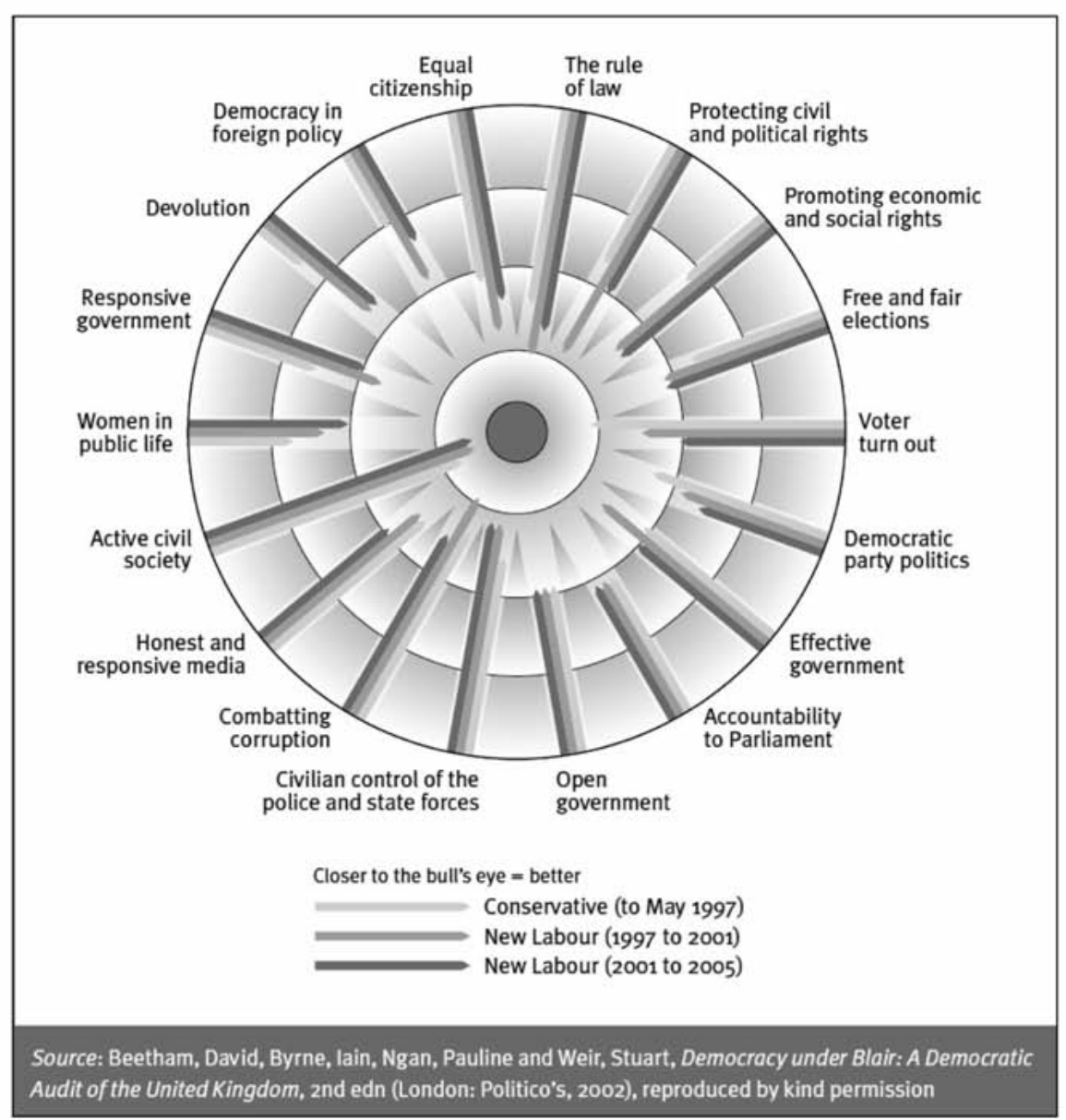

Als Vorbild für andere europäische Länder werden der in Schweden weitgehend freie Zugang zu öffentlichen Dokumenten (Transparenz) und das Modell der autonomen Verwaltungseinheiten betrachtet. ${ }^{21}$ Allerdings werden auch Probleme identifiziert: „The weak points are limited access to the political agenda, quality problems in the public sphere, intolerance, lack of clarity in the separation of powers, deficient accountability and weaknesses when it comes to economic

20 Siehe auch: The Democratic Audit, a.a.O., 3.

21 Petersson, O.: The Democratic Audit of Sweden, Stockholm, 2001, 13. 
resource control “.22 Als besonders heikel wird der Rückgang der Mitgliedschaft in politischen Parteien bewertet, der die öffentliche Kommunikation und die Findung von Lösungen, die auf breitem sozialem Konsens beruhen, erschwert. ${ }^{23}$

Einen bescheideneren Versuch, den Vorschlag von Beetham umzusetzen, bildet eine Fallstudie zu Österreich. ${ }^{24}$ In dieser Studie werden zwei Problematiken des Audit deutlich: (1) Die fehlende Explikation der Fragen und der Beurteilungsmaßstäbe führt zu einem ,innovativen“ Umgang mit der methodischen Vorlage. ${ }^{25}$ Als Bewertungsmaßstäbe werden etwa verschiedene Zeitperioden in der österreichischen Politik ausgewählt. ${ }^{26}$ Damit lassen sich zwar Angaben über Veränderungen machen, jedoch keine Aussagen treffen, welche Demokratiequalität der jeweilige Befund aufweist. Die assoziative Übernahme von Kriterien ist gleichfalls nicht unproblematisch, wie die Interpretationsbreite im Verständnis einzelner Fragen verdeutlicht. (2) Der Audit erfordert - nicht unbedingt eine Schwäche - ausgiebige empirische Forschungen, um die jeweiligen Einschätzungen hinreichend illustrieren und belegen zu können.

Generell verweisen die leicht divergierenden Umsetzungen des Konzepts zudem nochmals auf die Schwierigkeiten im komparativen Einsatz, der aber auch nur bedingt angestrebt wird, wie auf der Homepage konstatiert wird: ,the results of the assessments are therefore not comparable in the same ways as extant measures developed in mainstream political science ${ }^{27}{ }^{27}$ Die Vergleichsstudien haben zugleich darauf aufmerksam gemacht, dass die Ergebnisse zum Teil auch quantifiziert werden. Schließlich zeigen die Anmerkungen zu den empirischen Befunden, dass sich die Methodologie des Democratic Audit im Laufe der Zeit gewandelt hat und nun weitgehend dem Ansatz von IDEA entspricht.

22 Petersson, O., a.a.O., 2.

23 Erschienen sind gleichfalls Studien zur Demokratiebewertung in Irland (Hughes, I./Clancy, P./Harris, C./Beetham, D.: Power to the People. Assessing Democracy in Ireland, Dublin, 2007), Australien und den Niederlanden (Ministry of the Interior and Kingdom Relations: The Netherlands: The State of Our Democracy/De Staat van Onze Democratie, The Hague, 2006).

24 Campbell, D.F.J./Liebhart, K./Martinsen, K./Schaller, C./Schedler, A. (Hg.): Die Qualität der österreichischen Demokratie. Versuche einer Annäherung, Wien, 1996.

25 Gerlich, P.: Öffentlichkeit, Zugänglichkeit, Kontrolle. Zur demokratischen Qualität staatlicher Institutionen, in: Campbell, D.F.J./Liebhart, K./Martinsen, K./Schaller, C./Schedler, A. (Hg.), a.a.O., 45-58, hier $50-53$.

26 a.a.O., 55.

27 The Democratic Audit: Comparing Democracies. 


\section{International IDEA Framework}

Das International Institute for Democracy and Electoral Assistance (IDEA) ist eine intergouvernementale Organisation von rund 30 Staaten zur weltweiten Förderung der Demokratie mit Sitz in Stockholm. Unterstützt werden Demokratisierungsprozesse unter anderem durch Beratung bei Wahlen, Unterstützung beim Aufbau von Parteiorganisationen und im Bereich der Geschlechtergleichstellung. Noch stärker als andere Ansätze löst sich die Vorgehensweise der Demokratiemessung von der Bewertung externer Begutachter und legt es in die Hände ,integrer“ nationaler Forschungsteams unter Einbezug von Regierungsstellen und/oder Vertretern von Zivilgesellschaft und Wissenschaftseinrichtungen, die selbst auch als Adressat und Träger demokratischer Bildung begriffen werden. Intensiver als beim Democratic Audit steht die politische Ausrichtung auf Stärkung der Demokratie durch Reformmaßnahmen im Zentrum der Arbeit, wobei junge und etablierte Demokratien einbezogen werden. Die Forschungsanleitungen von IDEA sind speziell darauf ausgerichtet, die entsprechenden Evaluationskompetenzen zu vermitteln. Eine umfangreiche Publikationstätigkeit, in der auch die bisherigen Ergebnisse dokumentiert werden, unterstützt diesen Anspruch. $^{28}$

Der Ansatz von IDEA folgt in der Fokussierung auf popular control und political equality dem Demokratieverständnis des Democratic Audit und unterscheidet vier zentrale Untersuchungsfelder, die anhand von insgesamt 15 Leitfragen konkretisiert werden (vgl. Tab. 1). Durch die Einführung einer Zwischenebene (mediating values) erfolgt eine maßgeblich institutionell gelagerte Vermittlung zwischen der abstrakten Definitionsebene und den Leitfragen. Insgesamt werden hier sieben Kategorien unterschieden: Partizipation, Autorisierung (entspricht weitgehend freien und fairen Wahlen), Repräsentation, accountability, Transparenz, responsiveness und Solidarität. Auf der Ebene der Leitfragen hat der Ansatz deutlich an Komplexität verloren. Speziell die Bereiche open and accountable government und democratic society wurden gekürzt. Als eigenständige Kategorie neu aufgenommen wurde democracy beyond the state, in der sowohl nach externen Einflüssen auf die Entwicklung einer Demokratie gefragt wird als auch nach der Unterstützung von demokratischen Prozessen in anderen Ländern und der Akzeptanz von internationalen Rechtsstandards. ${ }^{29}$ Neu angefügt ist die

28 Vgl. unter www.idea.int.

29 Hierzu gehören z.B. folgende Fragen: 4.2.1. How consistent is the government in its support for, and protection of, human rights and democracy abroad? 4.2.2. How far does the government support the 
Frage nach den wirtschaftlichen und sozialen Rechten. Gleichfalls zum ersten Mal findet sich die Frage nach der zivilen Kontrolle von Militär und Polizei. Betont wird die Rolle des Parlaments, dessen Einflussverlust bei nationalen und internationalen Entscheidungen zu Bewertungsabstrichen führt. Die Straffung der Leitfragen wird durch die Einführung einer weiteren Ebene mehr als kompensiert, da hier eine Konkretisierung durch drei bis acht weitere Fragen vorgesehen ist. Insgesamt lässt sich somit eine Zunahme der Konzeptkomplexität feststellen. Zugleich eröffnet die vorgesehene Skalierung der Antwort auf fünf Stufen eine quantifizierende Darstellung der empirischen Befunde. Wie bereits der Democratic Audit versucht IDEA die Messung kontextsensibel zu gestalten. Hierzu werden die Bewertungsstandards der Geschichte des Landes, der regionale Praxis und den eigenen Prioritären angepasst.

An diesen Beispielen wird ersichtlich, dass die dem Konzept zugrunde liegende Demokratievorstellung im Vergleich zum Democratic Audit deutlich unpräziser ausfällt. Einerseits werden soziale und wirtschaftliche Komponenten einbezogen und andererseits die internationale Ebene deutlich erweitert, wobei auch nach dem Umfang demokratieförderlicher bzw. -hinderlicher Aktivitäten jenseits des eigenen politischen Systems gefragt wird. Die Erweiterung des Fragenspektrums erbringt nur scheinbar eine Präzisierung des Demokratieverständnisses, da der jeweilige Maßstab zur Beantwortung der Fragen unklar bleibt bzw. neben der Orientierung an internationalen und regionalen Standards den nationalen Forschungsgruppen überlassen wird. Diese „Freigabe“ in der Konkretisierung des Maßstabs der Demokratie erschwert nicht nur die Vergleichbarkeit der Ergebnisse, sondern lässt die Konturen der Demokratie letztlich noch unschärfer werden.

UN and agencies of international cooperation, and respect the rule of law internationally? Der internationale Aspekt war im Democratic Audit-Konzept bislang mit der Frage 18 deutlich enger gefasst: How far does any supra-national level of government meet the criteria of popular control and political equality, whether through national parliaments or through representative institutions of its own? 
Tabelle 1: IDEA - The Assessment Framework: An Overview

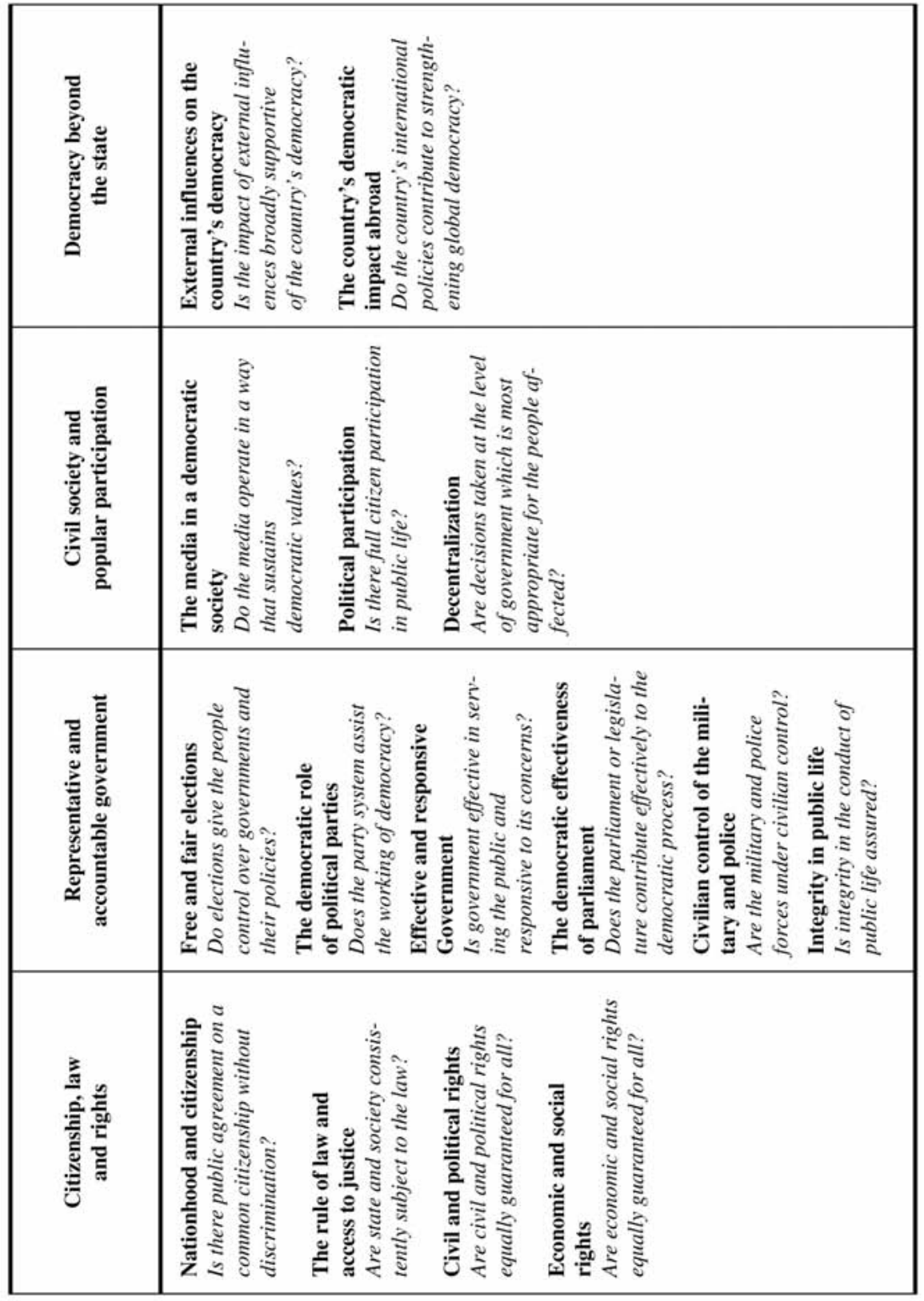

Quelle: Beetham, D./Carvalho, E./Landman, T./Weir, S.: Assessing the Quality of Democracy. A Practical Guide, Stockholm, 2008, 2. 
Generell unterscheiden IDEA und der Democratic Audit weder zwischen Merkmalen der Demokratie und den ihr dienlichen Faktoren noch zwischen ihrer Qualität und ihrer Konsolidierung. Dies mag für die politische Zielsetzung weniger störend sein, die wissenschaftliche Qualität aber leidet darunter. Weiterführend ist hingegen der Einbezug der internationalen Ebene, wenngleich auch hier die konzeptionelle Reflexion noch stärker zu systematisieren wäre. Dies betrifft sowohl die internationalen Einflüsse auf eine nationale Demokratie als auch deren externe Wirkung.

\section{Ein kontextualisiertes Maß für Demokratie}

Ein stärker komparativ ausgewiesener Vorschlag zur Demokratiemessung findet sich bei Heidrun Abromeit und, in der Weiterführung, Michael Stoiber. ${ }^{30}$ Abromeit geht dabei von der Grundannahme aus, dass es keinen übergreifenden institutionellen Maßstab der Demokratie geben kann, der für alle Staaten als Referenzrahmen dienen könnte. Vielmehr sei die jeweilige spezifische Gesellschaftsstruktur zu berücksichtigen, da sie unterschiedliche Anforderungen an die optimale Ausformung einer Demokratie bedingt. So sind beispielsweise in einem Land mit homogener Gesellschaftsstruktur andere Anforderungen an das Entscheidungsverfahren sowie an Wahl- und Parteiensystem zu stellen als in einem eher heterogen geprägten Gemeinwesen. Ähnlich hatte bereits Lijphart argumentiert, indem er homogenen Gesellschaften das Modell der Mehrheits- und heterogenen das der Konsensdemokratie empfahl. ${ }^{31}$

Das von Abromeit vorgeschlagene Demokratieverständnis ist schlank und konzentriert sich auf den Aspekt der effektiven Partizipation: ${ }^{32}$ „Demokratie ist die Verlängerung der individuellen Selbstbestimmung in den Bereich kollektiver Entscheidungen hinein; sie konkretisiert sich in der Beteiligung der Individuen an den Entscheidungen, von denen sie betroffen, denen sie unterworfen sind“. Aufgrund der engen Bindung an Entscheidungsverfahren sind die Basiskriterien

30 Abromeit, H.: Ein Maß für Demokratie. Europäische Demokratien im Vergleich. IHS Political Science Series No. 76, Wien, 2001; Abromeit, H.: Die Messbarkeit von Demokratie, a.a.O.; Stoiber, M.: Zur Messbarkeit von Demokratie. Konzeptualisierung und Operationalisierung eines kontextualisierten MaBes, in: TU Darmstadt, Institut für Politikwissenschaft, Working Papers Nr. 5, 2005; Stoiber, M.: Ein neues, kontextualisiertes Maß für Demokratie, a.a.O. Nicht berücksichtigt werden konnte die noch nicht erschienene Monographie: Stoiber, M: Die Qualität von Demokratien im Vergleich: Zur Bedeutung des Kontextes in der empirisch vergleichenden Demokratietheorie, Baden-Baden, 2011.

31 Lijphart, A.: Patterns of Democracy. Government Forms and Performance in Thirty-Six Countries, New Haven/London, 1999.

32 Abromeit, H.: Die Messbarkeit von Demokratie, a.a.O., 78. 
in Abromeits Vorschlag stark an Partizipationsmöglichkeiten ausgerichtet. Gefragt wird nach deren Umfang und Relevanz, ihrer Differenziertheit und Inklusion, wobei Minderheiten, territorialen Untergliederungen und sektorale Segmente besondere Beachtung erfahren.

Die Grundidee, den Kontext in der Messung einzubeziehen, ist plausibel. Allerdings trifft Abromeits Kritik an institutionell gelagerten Demokratiedefinitionen nur dann zu, wenn es sich um Demokratiedefinitionen handelt, die auf konkreten institutionellen Merkmalen basieren. Dies wäre der Fall, wenn sich der Maßstab der Demokratie an bestimmten institutionellen Konfigurationen (z.B. parlamentarisches Regierungssystem, Mehrheitswahlrecht, Mehrparteiensystem, direkte Demokratie) ausrichten würde. Allerdings ist dieser Einwand bei den gängigen Ansätzen der Demokratiemessung kaum mehr virulent; er trifft eher die älteren Vorschläge. ${ }^{33}$ Die Kritik geht zudem ins Leere, wenn eine Demokratiedefinition vorliegt, die sich auf zentrale Funktionsleistungen einer Demokratie bezieht. So ist die Aussage, dass das Partizipationssystem auf offenen, freien und fairen Verfahren beruht, ein allgemeiner Maßstab, der für jedes Land gelten sollte. Welche institutionellen Ausprägungen diesen Maßstab im Einzelnen umsetzen, ist damit noch nicht festgelegt. Es gibt durchaus unterschiedliche Institutionen, die geeignet sind; dabei ist die Möglichkeit funktionaler Äquivalente zu bedenken. Es ist dann jeweils im Einzelfall zu prüfen, inwieweit das spezifische institutionelle Ensemble die ihm zugedachten demokratischen Funktionsleistungen angemessen und effektiv zum Ausdruck bringen kann. So wird ihr Anliegen, dass sich eine Demokratie auf gleicher Qualitätsstufe in unterschiedlichen institutionellen Arrangements ausdrücken kann, bereits umfassend beachtet.

Interessanter ist der weitergehende Vorschlag, den Kontext systematisch in die Bewertung einzubeziehen, indem sie auf der Grundlage einer Länderanalyse bereits selbst das adäquate institutionell gelagerte $\mathrm{Ma} ß$ der Demokratie und somit das der Demokratie angemessene institutionelle Ensemble formulieren möchte. Dabei ist zu klären, in welchem Maße direktdemokratische oder repräsentative Instrumente angemessener sind. Hierzu wird der Kontext anhand von vier Variablen strukturiert: (1) Heterogenität einer Gesellschaft, (2) Dominanzstrukturen, (3) institutionelle Komplexität und (4) (In)formalisierung des Entscheidungssystems. Michael Stoiber hat es unternommen, diese Kontextvariablen zu operationalisieren und in quantitative Maße zu überführen, die es letztlich ermöglichen,

33 Vgl. die Operationalisierung in Dahl, R.A.: Polyarchy. Participation and Opposition, New Haven/London, 1971. 
ein Maß der Demokratie zu bestimmen, das den Bedarf an Beteiligungsarten mit der Existenz von gegebenen Partizipationsmöglichkeiten abgleicht (vgl. Abb. 2). Das Ausmaß und die spezifische Kombination der Kontextvariablen definieren den Bedarf an einer je eigenen Zusammensetzung der Beteiligungsarten. Hierbei wird zwischen Autonomie- und Vetorechten, direktdemokratischen Verfahren und differenzierten Beteiligungsstrukturen unterschieden. Die effektive Realisierung des optimalen Angebots entspricht dem Höchstwert der Messung (= 1); Abweichungen werden als Defizite interpretiert. Die Effektivität der Beteiligungsstruktur (Angebot) wird dabei unter anderem durch die Multiplikation der Wirksamkeit (Angemessenheit) der demokratischen Partizipationsformen mit dem Grad der Dominanzstruktur ermittelt; weitere Faktoren sind die Einschränkung direktdemokratischer Entscheidungen, die Existenz bedeutungsvoller Wahlen, das Ausmaß indirekter Wahlverfahren und die Wirksamkeit von Minderheitenrepräsentation.

Zentral für die Messung sind theoretisch begründete Annahmen über den Zusammenhang zwischen den Kontextvariablen und dem jeweiligen Bedarf, deren Ergebnisse sich beispielhaft in folgenden Aussagen sich ausdrücken: ${ }^{34}$ Jegliche Art an Informalisierung erhöht den Bedarf an direktdemokratischer Beteiligung. Bei einer Segmentierung der Gesellschaft ist die Anwendung der Mehrheitsregel inadäquat. Gefordert sind hier qualifizierte Mehrheiten oder bei äußerst sensiblen Entscheidungen Veto- oder Autonomierechte. In Mehrebenensystemen sind differenzierte Entscheidungssysteme, die eine effektive Beteiligung auf allen Ebenen ermöglichen, angemessen. Je ausgeprägter Dominanzstrukturen sind, desto stärker werden die Präferenzen der Bürger verzerrt. Die einzelnen Variablen werden in der Operationalisierung spezifiziert, wobei in diesem Forschungsschritt die damit verbundene Inhaltspräzisierung nicht näher theoretisch reflektiert wird. So werden die Dominanzstrukturen mit folgenden drei Indikatoren erfasst: „partyness “ of society (Ausmaß der Verknüpfung von relevanten Parteien und großen gesellschaftlichen Akteuren), Versäulung oder Lagerbildung (Stärke politischer Subkulturen und Elitenverbindungen) und Mediensystem (Monopolstruktur). ${ }^{35}$ Die Verbindung dominanter sozialdemokratischer Parteien mit Gewerkschaften wird hiermit analog der Ausprägung monopolartiger Medienstrukturen gesetzt.

34 Stoiber, M.: Zur Messbarkeit von Demokratie, a.a.O., $11 \mathrm{f}$.

35 Ebd., 18. 
Abbildung 2: Kontextvariable und Beteiligungsarten

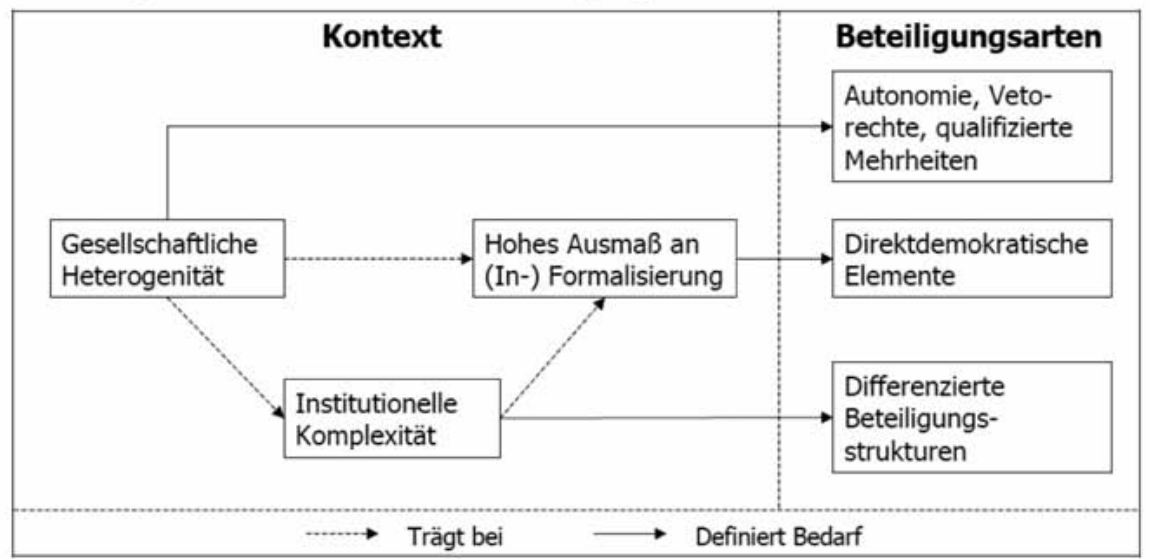

Quelle: Stoiber, M.: Zur Messbarkeit von Demokratie, a.a.O.,11.

Die Messanlage ist komplex angelegt und bedarf einer umfangreichen Datenermittlung in zweierlei Hinsicht. Zum einen erfordert die Benennung des jeweiligen Bedarfs an Institutionen eine exakte Analyse. Zum anderen ist das jeweilige Beteiligungsangebot in seiner effektiven Realisierung zu bestimmen. Insgesamt erweisen sich viele subjektive Einschätzungen als notwendig, um die entsprechenden Indikatoren zu bearbeiten. Das Hauptproblem liegt jedoch in der Grundannahme, dass es für jedes politische System eine einzige angemessene institutionelle Lösung der Beteiligungsstruktur geben soll. So werden mögliche funktionale Äquivalente ausgeblendet. Daher wäre zu diskutieren, inwieweit der jeweils aufgestellte kontextspezifische Maßstab überzeugen kann, der nun eine weitaus größere theoretische Beweislast zu erbringen hat, indem er für jedes Land ein eigenes spezifisches Optimum formulieren möchte. Diese Problematik wird dadurch verstärkt, dass einige institutionelle Merkmale nun wiederum tendenziell als Maß gesetzt werden, wie die Präferenz für föderalistische Strukturen gegenüber unitarischen Ordnungen zeigt. Auch die Validität der Operationalisierung ist nicht überall gegeben. Dies zeigt sich in der Messung der Informalität. ${ }^{36}$ Nicht überraschend ist hier, dass für Österreich und die Schweiz hohe Werte gemessen werden; dagegen ist es durchaus kontraintuitiv, dass der höchste Wert der Informalität für Schweden festgestellt wird, während Italien und Großbritannien die niedrigsten Werte verzeichnen. Dieser Befund ist nicht plausibel und zeigt, dass in der Operationalisierung eine korporatistische Variable zum Einsatz 
kommt, während informelle Praktiken oder informelle Institutionen überhaupt nicht einbezogen werden. ${ }^{37}$

Ein anderer Kritikpunkt betrifft das bereits angesprochene schlanke Demokratieverständnis, das nur auf effektive Partizipationsmöglichkeiten ausgerichtet ist. Nicht berücksichtigt werden somit alle Mechanismen der horizontal accountability und der Rechtsstaatlichkeit, deren Bedeutung für die Demokratie in der Auseinandersetzung mit dem Polyarchiemodell von Dahl mit guten Gründen aufgezeigt wurde. ${ }^{38}$ Ebenfalls weitgehend ausgeblendet werden Kommunikations- und Organisationsfreiheiten. Dass das Maß der Freiheit und Fairness von Wahlen nicht systematisch in der Operationalisierung erfasst wird, ist dagegen eher als Validitätsproblem zu werten, da dieser Aspekt in der Demokratiedefinition durchaus enthalten ist. Zudem bezieht sich die Operationalisierung überwiegend auf institutionelle und strukturelle Faktoren; der faktische Umgang mit diesen Regeln durch die beteiligten Akteure wird nur verkürzt dargestellt. So muss gefragt werden: Was wird überhaupt gemessen? Es ist jedenfalls nicht die Qualität der Demokratie in einem umfassenden Sinne. Erfasst werden systematisch Beteiligungsformen, deren demokratische Qualität aus den genannten Gründen jedoch nur partiell ermittelt wird. Gleichwohl eröffnen die empirischen Studien auf dieser Grundlage einen differenzierten Einblick in die Vielfalt von Demokratien und erlauben durchaus, unterschiedliche Profile von Demokratie deutlich zu machen. Das dabei eingesetzte Messverfahren kann jedoch nicht restlos überzeugen.

\section{Die Demokratiematrix}

Analog der Konzeption des Democratic Audit bietet Lauth schließlich einen umfassenden Vorschlag für eine systematische Demokratiemessung. ${ }^{39}$ Die Grundlage bildet ein umfangreich erläutertes Demokratieverständnis, das durch die Dimensionen von politischer Freiheit, politischer Gleichheit und politischer und rechtlicher Kontrolle definiert wird und in fünf zentralen Institutionen ihren Ausdruck findet: Entscheidungsverfahren, intermediäre Vermittlung, öffentliche

37 Lauth, H.J.: Informal Institutions and Democracy, in: Democratization Vol. 7, No. 4 (Winter) 2000, 2150.

38 O'Donnell, G.: Why the Rule of Law matters, in: Journal of Democracy, 15/4 (2004), 32-46; Merkel, W.: Systemtransformation: Eine Einführung in die Theorie und Empirie der Transformationsforschung, 2. Aufl., Wiesbaden, 2010; Lauth, H.-J.: Demokratie und Demokratiemessung, a.a.O.

39 Lauth, H.-J.: Demokratie und Demokratiemessung, a.a.O. 
Kommunikation, Rechtsgarantie, Regelsetzung und Regelanwendung. In der Kombination beider Kategorien ergibt sich eine 15-Felder-Matrix (vgl. Abb. 3). Die Funktionsleistungen der Institutionen werden für alle Felder beschrieben. Sind diese in voller Ausprägung gegeben, so gilt der Maßstab der Bewertungskategorie 1 als erfüllt. Am anderen Ende der Skala findet sich die Kategorie 5, die greift, wenn bei der Untersuchung der Variablen keine ausreichenden Werte erzielt werden. Die Bewertungen reichen somit in jedem Feld von eins (beste Einschätzung) bis fünf (nicht mehr demokratisch); die Ergebnisse zwischen eins und drei liegen im Bereich einer funktionierenden Demokratie und der Wert vier kennzeichnet ein System, das durch institutionelle Defizite geprägt ist. ${ }^{40}$ Drei Regeln sind bei der Regimeklassifikation zu beachten, wobei sie nur zum Einsatz kommen können, wenn für alle 15 Matrixfelder Messwerte vorliegen:

- Regel 1: Sobald ein Matrixwert als „nicht ausreichend“ eingestuft wird, kann der Gesamtbefund nicht mehr dem Bereich der Demokratien zugeordnet werden. Reziprok gilt, dass eine Demokratie vorliegt, wenn alle Messwerte mindestens , ausreichend“ aufweisen.

- Regel 2: Sobald ein Matrixwert in die Kategorie „,ausreichend“ fällt, wird das Regime als defizitäre Demokratie bezeichnet. Dies setzt allerdings voraus, dass kein Matrixwert als „nicht ausreichend“ eingestuft wird.

- Regel 3: Sobald kein Matrixwert geringer als „befriedigend“ eingestuft wird, liegt eine funktionierende Demokratie vor.

Die Matrix erlaubt somit eine differenzierte Profilzeichnung einer Demokratie, in der die Stärken und Schwächen nach Institutionen und Dimensionen gekennzeichnet werden. Darüber hinaus ermöglicht sie die Unterscheidung von Regimetypen.

Die Untersuchung der anhand von Rechten und Regeln beschriebenen Funktionsleistungen erfolgt auf vier Ebenen: (1) Existenz der Rechte (Kodifizierung), (2) Praktizierungsmöglichkeit der Rechte im Sinne ihrer administrativen und institutionellen Ausstattung und (3) Verhinderung der Rechte durch formale oder informelle Eingriffe (Ausmaß an Rechtsverletzung). Nicht überprüft wird der Umfang der Anwendung von Rechten, da diese Strategie - wie das Beispiel der Wahlbeteiligung zeigt - eine Reihe von Problemen in sich birgt. Die gleiche Überlegung gilt generell für die Wirksamkeit von Regeln, wenn sie ermöglichender Natur sind. Haben sie jedoch wie bei Gesetzen einen verpflichtenden Charakter, so ist die Nicht-Beachtung der Regel als Indikator zu nehmen. Dem

40 Nähere Erläuterungen in Lauth, H.-J.: Demokratie und Demokratiemessung, a.a.O. 
folgend und abweichend von der Strategie der Erfassung der Freiheits- und Gleichheitsdimension erfolgt die Messung der Kontrollrechte. Hier muss ebenfalls gefragt werden, ob diese praktiziert werden. Denn der Nachweis fehlender Restriktionen belegt noch nicht die Existenz einer funktionierenden aktiven Kontrolle. Hier ist in einer vierten Ebene (4) zu prüfen, inwieweit die Kontrollrechte wahrgenommen werden.

Abbildung 3: Die 15-Felder-Matrix der Demokratie: Möglichkeiten der Indexbildung

\begin{tabular}{|c|c|c|c|c|}
\hline$\underbrace{\text { Dimensionen }}_{\text {Institutionen }}$ & Freiheit & Gleichheit & Kontrolle & $\begin{array}{l}\text { Index- } \\
\text { bildung }\end{array}$ \\
\hline $\begin{array}{c}\text { Entscheidungs- } \\
\text { verfahren }\end{array}$ & $\begin{array}{l}\text { Freie Wahlen und } \\
\text { Plebiszite } \\
01 \quad 1 / 1 \\
\end{array}$ & $\begin{array}{l}\text { Gleiche Partizi- } \\
\text { pationschancen, } \\
\text { Stimmengleichheit } \\
06 \quad 1 / 2 \\
\end{array}$ & $\begin{array}{l}\text { Kontrolle durch } \\
\text { Wahlkommission } \\
11 \quad 1 / 3 \\
\end{array}$ & Instindexl \\
\hline $\begin{array}{l}\text { Intermediäre } \\
\text { Vermittlung }\end{array}$ & $\begin{array}{l}\begin{array}{l}\text { Organisations- } \\
\text { freiheiten }\end{array} \\
02 \quad 2 / 1\end{array}$ & $\begin{array}{l}\text { Gleiche Organi- } \\
\text { sations- und Hand- } \\
\text { lungsrechte } \\
07 \quad 2 / 2\end{array}$ & $\begin{array}{l}\text { Kontrolle durch } \\
\text { Verbände, Parteien, } \\
\text { Zivilgesellschaft } \\
12 \quad 2 / 3\end{array}$ & Instindex 2 \\
\hline $\begin{array}{c}\text { Kom- } \\
\text { munikation/ } \\
\text { Öffentlichkeit }\end{array}$ & $\begin{array}{ll}\begin{array}{l}\text { Kommunika- } \\
\text { tionsfreiheiten }\end{array} \\
\\
03 & 3 / 1 \\
\end{array}$ & $\begin{array}{l}\text { Gleiche } \\
\begin{array}{l}\text { Beteiligungs- } \\
\text { chancen }\end{array} \\
08\end{array}$ & $\begin{array}{l}\text { Kontrolle durch } \\
\begin{array}{l}\text { Medien } \\
\text { (unabhängiger } \\
\text { Journalismus) }\end{array} \\
13 \quad 3 / 3 \\
\end{array}$ & Instindex 3 \\
\hline Rechtsgarantie & $\begin{array}{l}\text { Freier Zugang zur } \\
\text { Justiz } \\
04 \quad 4 / 1 \\
\end{array}$ & $\begin{array}{l}\text { Gleiche Rechte und } \\
\text { gleiche Behandlung } \\
\text { durch Justiz } \\
09\end{array}$ & $\begin{array}{l}\text { Effektive Recht- } \\
\text { sprechung, Ver- } \\
\text { fassungsgericht } \\
14 \quad 4 / 3 \\
\end{array}$ & Instindex4 \\
\hline $\begin{array}{l}\text { Regelsetzung } \\
\text { und Regel- } \\
\text { anwendung }\end{array}$ & $\begin{array}{l}\text { Effective govern- } \\
\text { ment (Parlament, } \\
\text { rationale Büro- } \\
\text { kratie) } \\
05\end{array}$ & $\begin{array}{l}\text { Gleiche Behandlung } \\
\text { durch Parlament } \\
\text { und Verwaltung } \\
10 \quad 5 / 2 \\
\end{array}$ & $\begin{array}{l}\text { Gewaltenteilung } \\
\text { (Parlament. Opposi- } \\
\text { tion, zweite Kam- } \\
\text { mer, Rechnungshof) } \\
15\end{array}$ & Instindex 5 \\
\hline Indexbildung & DimexlF & Dimex $2 G$ & Dimex $3 K$ & $\begin{array}{l}\text { Gesamt- } \\
\text { index } \\
\text { (Demex) }\end{array}$ \\
\hline
\end{tabular}

Quelle: Eigene Darstellung nach Lauth, H.-J.: Demokratie und Demokratiemessung, a.a.O., 330. 
Die Beurteilungen umfassen jeweils ein Jahr. Wenn nicht - wie etwa im Falle von Wahlen - spezifische Zeitpunkte der Messung vorliegen, sind bei jeder Beurteilung die Ereignisse des gesamten Jahres zu berücksichtigen. Es ist dabei zu beachten, dass bereits für die Einschätzung einfacher Indikatoren eine Fülle von Beobachtungen vorliegt, die zu einem Wert aggregiert werden. Eine mögliche Gewichtung der Befunde wird durch die jeweilige Begründung der Einstufung implizit geliefert. Zwei Varianten der Messung werden vorgeschlagen: Eine Vergleichsstudie, die mit „schlanken Indikatoren“ arbeitet, und eine zweite Variante, die auf eine umfassende Messung aller Indikatoren zielt.

Die Relevanz des Kontextes wird über die funktionale Beschreibung der Institutionen erfasst. Demnach erfüllen die Institutionen der einzelnen Matrixfelder spezifische Funktionen, die auf einem universellen Anspruch gründen. Erst in der empirischen Prüfung wird untersucht, welche konkreten Institutionen diese Funktionen wahrnehmen. Hierbei können unterschiedliche institutionelle Ausprägungen die gleiche Funktion wahrnehmen. Zwar ist der Blick durch die Kenntnis bestehender demokratischer Einrichtungen geschärft, doch bleibt er offen für die Identifizierung funktionaler Äquivalente. Berücksichtigt wird somit der Sachverhalt, dass gleiche Institutionen in divergierenden Kontexten unterschiedliche Wirkungen haben können und dass länderspezifische Institutionen (auch informeller Art) in die Prüfung funktionaler Äquivalenzleistungen einbezogen werden.

Eine fundierte wissenschaftliche Anwendung liegt hier für diese Messanlage bislang nur für Fallstudien vor. ${ }^{41}$ Der Befund der Fallstudie von Österle, die mit dem schlanken Indikatorenset arbeitet, ist eindeutig. ${ }^{42}$ Das politische System von Russland hat unter Putin in vielen Bereichen das demokratische Spektrum verlassen und kann nicht mehr als Demokratie bezeichnet werden (vgl. Abb. 4). Da jedoch noch einige Felder im demokratischen Spektrum liegen, kann es als hybrides Regime verstanden werden. Die Schwachstellen liegen in den Institutionen „Kommunikation und Öffentlichkeit“ sowie „Rechtsgarantie“. Entlang der dimensionalen Achse ist speziell die Kontrolle schwach ausgeprägt. Patze, der mit seiner leichten Veränderung der Messanlage die Befunde stärker akzentuiert, spricht sogar von einem stark autoritären politischen System. ${ }^{43}$

41 Österle, I.: Wohin entwickelte sich die Qualität der Demokratie in Russland? - Ein Vergleich der Amtszeiten der Präsidenten Jelzin und Putin, Würzburg, 2009; Patze, P.: Wie demokratisch ist Russland? Ein tiefenorientierter Ansatz zur Messung demokratischer Standards, Baden-Baden, 2011.

42 Österle, I., a.a.O.

43 Patze, P., a.a.O., 311. 
Abbildung 4: Demokratiematrix: Russland unter Putin (schlanke Indikatoren)

\begin{tabular}{|c|c|c|c|c|}
\hline & Freiheit & Gleichheit & Kontrolle & $\Sigma / \varnothing$ \\
\hline $\begin{array}{l}\text { Entscheidungs- } \\
\text { verfahren }\end{array}$ & $\begin{array}{c}\text { Freie nationale } \\
\text { Wahlen } \\
\\
3\end{array}$ & $\begin{array}{c}\text { Gleiches aktives } \\
\text { und passives } \\
\text { Wahlrecht } \\
3\end{array}$ & $\begin{array}{c}\text { Unabhängige } \\
\text { Wahlprüfungs- } \\
\text { kommission } \\
4\end{array}$ & $11 / 3,3$ \\
\hline $\begin{array}{l}\text { Intermediäre } \\
\text { Vermittlung }\end{array}$ & $\begin{array}{l}\text { Organisationsfrei- } \\
\text { heit für Parteien } \\
\text { und Gewerk- } \\
\text { schaften } \\
3\end{array}$ & $\begin{array}{c}\text { Faire Parteienfi- } \\
\text { nanzierung und } \\
\text { Konsultationsme- } \\
\text { chanismen } \\
4 \\
\end{array}$ & $\begin{array}{c}\text { Politische } \\
\text { Kontrolle durch } \\
\text { Oppositions- } \\
\text { parteien } \\
5 \\
\end{array}$ & $12 / 4,0$ \\
\hline $\begin{array}{l}\text { Kommunikation } \\
\text { Öffentlichkeit }\end{array}$ & $\begin{array}{c}\text { Freie Presse } \\
5 \\
5\end{array}$ & $\begin{array}{l}\text { Vielfalt der Me- } \\
\text { dienlandschaft } \\
5 \\
\end{array}$ & $\begin{array}{l}\text { Regierungskriti- } \\
\text { sche Presse } \\
5 \\
\end{array}$ & $15 / 5,0$ \\
\hline Rechtsgarantie & $\begin{array}{c}\text { Unabhängigkeit } \\
\text { der Justiz }\end{array}$ & $\begin{array}{l}\text { Rechtssicherheit } \\
\text { für marginale } \\
\text { Gruppen } \\
\\
5 \\
\end{array}$ & $\begin{array}{c}\text { Effektive Recht- } \\
\text { sprechung gegen- } \\
\text { über anderen } \\
\text { staatlichen } \\
\text { Institutionen } \\
4 \\
4\end{array}$ & $14 / 4,6$ \\
\hline $\begin{array}{l}\text { Regelsetzung } \\
\text { und } \\
\text {-anwendung }\end{array}$ & $\begin{array}{c}\text { Staatlichkeit und } \\
\text { das Fehlen von } \\
\text { tutelary powers } \\
3 \\
\end{array}$ & $\begin{array}{l}\text { Ausmaß der } \\
\text { Korruption in der } \\
\text { Verwaltung } \\
4 \\
\end{array}$ & $\begin{array}{l}\text { Wirksame Rech- } \\
\text { nungshöfe und } \\
\text { parlamentarische } \\
\text { Kontrollrechte } \\
4 \\
\end{array}$ & $11 / 3,6$ \\
\hline$\Sigma / \varnothing$ & $19(3,8)$ & $21(4,2)$ & $22(4,4)$ & $62 / 4,1$ \\
\hline
\end{tabular}

Quelle: Erweiterte Darstellung nach Österle, I., a.a.O., 94.

Ein Vergleich der Amtszeiten der beiden Präsidenten Jelzin und Putin kann gut die Veränderungen weg von einer defizitären Demokratie (Jelzin) in den Bereich der Autokratie gut verdeutlichen (vgl. Abb. 5). Mit Ausnahme eines einzigen Matrixfeldes stagnierte die Demokratiequalität oder hat sich vor allem in der Dimension der Freiheit und Kontrolle stark verschlechtert. Die positive Entwicklung betrifft das Feld „Staatlichkeit und das Fehlen von tutelary powers“, sie reflektiert den Rückgewinn staatlicher Lenkungsmacht unter Putin. 
Abbildung 5: Vergleich der Demokratiequalität von Jelzin zu Putin

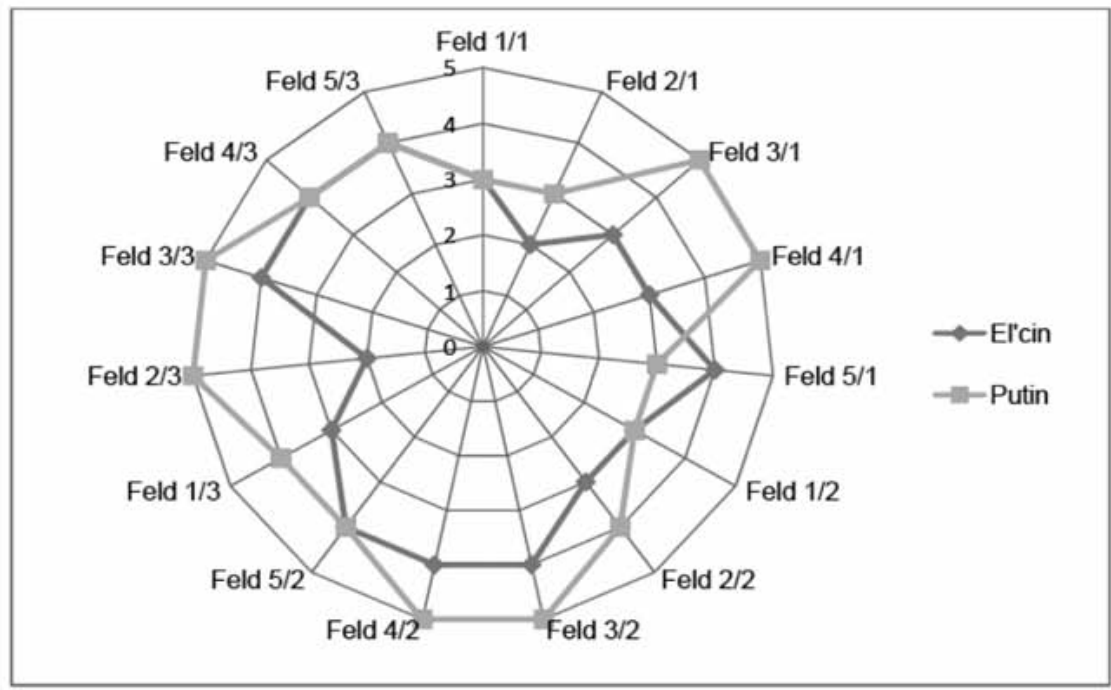

Quelle: Österle, I., a.a.O., 97.

Zugleich können andere vorläufige Ergebnisskizzen zeigen, dass auf diesem Wege ein differenziertes Profil auch von etablierten Demokratien zu erhalten ist. Hierbei zeigt sich, dass selbst etablierte Demokratien nicht vor regressiven Tendenzen geschützt sind. Braml und Lauth verdeutlichen die Probleme am Fall der USA, die im Kampf gegen den Terrorismus demokratische Qualitäten einbüßten. ${ }^{44}$ Ein weiteres, bislang noch nicht dokumentiertes Beispiel bietet Italien. Zwar ist der Gesamtindex für das Jahr 2004 mit 2,5 im Bereich der funktionierenden Demokratie angesiedelt. Doch der aggregierte Wert verdeckt die deutliche Profilbildung, die ihr zugrunde liegt. Demnach sind die Werte hinsichtlich der Institutionen Entscheidungsverfahren - maßgeblich Wahlen - und der intermediären Vermittlung (Organisationsfreiheiten) in allen Dimensionen im guten Bereich. Demgegenüber werden die Befunde zur öffentlichen Kommunikation deutlich schlechter bewertet. Als problematischstes Feld wird hierbei die Ausübung der Kontrollfunktion gesehen, da alle maßgeblichen TV-Sender - private und öffentliche - direkt dem Ministerpräsidenten Berlusconi unterstanden, der diese Position auch nachweislich nutzte, um Kritik an seiner Regierung zu unterbinden. Der Wert liegt hier im Bereich der defizitären Demokratie und ordnet

44 Braml, J/Lauth, H.-J:: The United States of America - A Deficient Democracy, in: Erdmann, G./Kneuer, M. (Hg.): Regression of Democracy, Wiesbaden, 2011 (i.E). 
nach den Klassifikationsregeln dieses Ansatzes Italien dem gleichnamigen Regimetypus zu. Auch nur zufriedenstellend wird die Rechtsgarantie eingeschätzt, wobei das Verhältnis von Politik und Recht ebenso kritische Fragen aufwirft wie der Einfluss von Mafia und Korruption. An dieser Stelle wurde der Bedarf an tiefer gehenden empirischen Forschungen besonders deutlich. Die Regelsetzung und -anwendung ließ keine gravierenden Mängel erkennen. Insgesamt wird die Freiheitsdimension besser als die Gleichheits- und Kontrolldimension bewertet.

Die Demokratiematrix steht mit ihrer Definition in relativer Nähe zum Bertelsmann-Transformationsindex (BTI) und zur Demokratieforschung des National Center for Competence in Research (NCCR), verfolgt aber eine andere Strategie in der konzeptionellen Entfaltung. Erste Einsätze in der Forschungspraxis hat die prinzipielle Tauglichkeit nachgewiesen. Es bleiben aber auch hier noch weitere Aspekte zu klären, die der empirischen Umsetzung zugewiesen werden. Der erste Punkt betrifft die Aggregation der Befunde pro Matrixfeld. Dies ist keine einfache Aufgabe, da stets eine Reihe von Indikatoren zu beachten ist, wobei die Befunde zudem räumlich und zeitlich variieren können. Zugleich kann jede Veränderung der Quellenlage zur Korrektur der Befunde führen. Zu beachten ist vor allem die Repräsentativität der empirischen Beobachtungen. Ein weiterer Punkt betrifft die Definition der Schwellenwerte. Wann genau ist die Zuordnung zu welcher Skalenstufe valide? Zentral soll dies durch die klare Bezeichnung des Maßstabes ermöglicht werden; eventuell kann dazu die weitere Präzisierung der vierten Skalenebene (defizitäre Ausprägung der Demokratie) auf theoretischer Ebene beitragen. Letztlich sind aber auch Erfahrungen im komparativen Umgang mit den Daten hilfreich. Zur Absicherung der Ergebnisse ist die Überprüfung der intercoder-Reliabilität ebenso zu beachten wie die transparente Dokumentation der Quellen und deren Interpretation. Der Einsatz der Demokratiematrix kann auf Grundlage des schlanken Indikatorensets oder durch eine umfassende Gesamterhebung erfolgen. Praktikabel ist aber auch die von Peter Patze vorgeschlagene Ausarbeitung, in der die 15 Matrixfelder zunächst durch zwei Indikatoren operationalisiert werden. Diese nun insgesamt 30 Subindikatoren werden in einem weiteren Schritt durch zusätzliche Fragen erläutert, so dass schließlich mehr als 60 Indikatoren zum Einsatz kommen. ${ }^{45} \mathrm{Zu}$ diskutieren wäre in dieser Variante allerdings die vorgeschlagene dichotome Messung. 


\section{Alternative: Umfragen als Messgrundlage?}

Wenngleich Daten der politischen Kulturforschung nur begrenzt Eingang in die Ansätze zur Demokratiemessung finden, wird oft diskutiert, ob nicht auch survey-Daten über Einstellungen zur Demokratie, ihren Institutionen und deren Effizienz sinnvoll zur Bestimmung der Qualität eingesetzt werden können. ${ }^{46}$ Dieses Anliegen ist nicht überraschend, wenn wir den Entstehungskontext der politischen Kulturforschung betrachten. Ein wichtiges Forschungsmotiv bestand in der Untersuchung der Stabilität von Demokratie - einem Aspekt, der in seiner Fixierung am Leitbild der civic culture ${ }^{47}$ bis heute in der Forschung zur Konsolidierung von Demokratien eine zentrale Rolle spielt. ${ }^{48}$ Das damit verbundene Erkenntnisinteresse thematisiert die Verankerung demokratischer Wertvorstellungen in der Bevölkerung, das Ansehen der demokratischen Institutionen, die Zufriedenheit der Bürger mit diesen und die staatsbürgerlichen Kompetenzen der Bürger selbst. Stabilität und Qualität einer Demokratie sind jedoch konzeptionell zu trennen. Wäre Stabilität ein konstitutives Merkmal einer Demokratie, könnten junge Demokratien - per definitionem - nicht die höchsten Werte erzielen. Von einer stabilen und qualitativ hochwertigen Demokratie zu sprechen, heißt von einer konsolidierten Demokratie zu sprechen. Die Stabilität fügt demnach der Qualität ein Merkmal hinzu. Können Messkonzepte, die in der politischen Kulturforschung zur Bewertung der Demokratiestabilität entwickelt wurden, für die Bestimmung der Qualität einer Demokratie verwendet werden? Diese Frage ist differenziert zu beantworten.

Begrenzt aussagekräftig erscheinen diejenigen Fragen, die auf die Anerkennung oder Ablehnung von demokratischen Werten zielen, da diese oftmals aus anderen Motiven genährt werden als durch die aktuellen Erfahrungen mit der zu beurteilenden Demokratie. Wieso sollte dann aber von diesen grundlegenden Einstellungswerten auf die aktuelle Qualität einer Demokratie geschlossen werden? Prinzipiell können auch in funktionierenden Demokratien die Anerkennungswerte divergieren. Die Messung der Qualität einer Demokratie betrachtet Handlungen und nicht Handlungsmotive. Mehr Aussagekraft scheinen Fragen zu haben, die sich mit der Zufriedenheit mit politischen Institutionen befassen. Nicht ohne

46 So auf dem Panel 'Measuring the Quality of Democracy on the Basis of Survey Data' im Rahmen einer Konferenz der European Survey Research Association (ESRA) in Prag (25-29 June 2007).

47 Almond, G.A./Verba, S.: The Civic Culture, Boston, 1965.

48 Diamond, L.: Developing Democracy. Toward Consolidation, Baltimore/London, 1999; Merkel, W., a.a.O. 
Grund liegt hier eine bessere Korrelation mit den ,quasi-objektiven“ Demokratiemessungen vor. ${ }^{49}$ Allerdings bestehen auch in diesem Fall Zweifel, ob die Messungen eine valide Darstellung der demokratischen Qualität ermöglichen. Folgende Einwände sind zu beachten: Die Vorstellungen von der Demokratie, ihren Werte und Institutionen divergieren innerhalb der Bevölkerung; folglich liegt der Beurteilung kein einheitlicher Maßstab zugrunde. Es kann kaum angenommen werden, dass (vor allem in jungen Demokratien) innerhalb einer Bevölkerung zu den relevanten Begriffen eine kohärente und gemeinsame Vorstellung existiert, geschweige denn im internationalen Vergleich. ${ }^{50}$ Mit diesem kulturellen bias kann durchaus die Zufriedenheit mit einer Institution oder einer Praxis einhergehen, die nach „objektiven“ demokratietheoretischen Maßstäben eher problematisch wäre, wie die Akzeptanz des Verzichts auf eine geschriebene Verfassung in Großbritannien zeigt. Aber selbst wenn die Begriffsverwendungen übereinstimmen würden, müsste dies nicht für die Erwartungen an die Performanz der Institutionen gelten. Doch gerade der bestehende Erwartungshorizont ist eine maßgebliche Folie für die Einschätzung der aktuellen Zufriedenheit, die wiederum als Resonanzboden für die Qualitätseinschätzung dient. ${ }^{51}$

Was besagt dieser Befund? Zunächst scheint es überraschend, dass die Einstellung der grundlegenden Akteure (der Staatsbürgerinnen und Staatsbürger) einer Demokratie wenig über deren Qualität aussagen kann. Ist ein solcher Standpunkt nicht Ausdruck einer Arroganz gegenüber dem demokratischen Souverän und einer Ignoranz gegenüber seiner Erfahrungswelt? Dies ginge wohl zu weit. Denn zum einen können den Staatsbürgern nicht alle für eine angemessene Einschätzung notwendigen Daten zur Verfügung stehen und zum anderen ergeben sich viele Bedenken aus einer komparativen Perspektive, die einen einheitlichen Bewertungsmaßstab erfordert. Schließlich ist noch zu beachten, dass Umfragedaten durchaus eine Rolle für die Qualitätsmessung spielen. Ein wichtiger Aspekt

49 Pickel, G.: Subjektive und objektive Indikatoren der Demokratiemessung im Vergleich - Grundlegende Unterschiede oder gleiche Ergebnisse?, in: Lauth, H.-J./Pickel, G./Welzel, C. (Hg.): Demokratiemessung. Konzepte und Befunde im internationalen Vergleich, Opladen, 2000, 242-265.

50 So liegt beispielsweise die positive Bewertung der demokratischen Performanz in Aserbaidschan noch vor Norwegen und weit vor den Demokratien Westeuropas (Klingemann, H.-D.: Unterstützung für die Demokratie. Eine globale Analyse für die 1990er Jahre, in: Lauth, H.-J./Pickel, G./Welzel, C. (Hg.): Demokratiemessung, Konzepte und Befunde im internationalen Vergleich, Opladen, 2000, 266-297, hier 290ff.). Die begrenzte Aussagekraft liegt auf der Hand.

51 Ein Beispiel für eine mögliche Diskrepanz in der Einschätzung der Demokratie bilden politische Skandale. Während damit in den Augen der Bevölkerung oftmals eine Abwertung der Qualität der Demokratie verbunden ist, können gerade das Aufdecken eines Skandals und seine Bearbeitung Anzeichen für die Qualität der Demokratie (etwa als wirksame Kontrolle) sein. 
von Umfragen ist die kognitive Ebene der politischen Kulturmessung. Die damit einbezogenen Ergebnisse könnten nicht nur als Reflexionsboden für die Einschätzung der evaluativen und affektiven Befunde dienen, sondern lassen sich auch als direkte Möglichkeit nutzen, die Qualität der Demokratie zu bestimmen, indem sie darüber informieren, inwieweit die Rechte, Verfahren und Institutionen der Demokratie den Bürgern bekannt sind. Darüber hinaus könnten Umfragen ähnlich wie beim Democratic Audit die Wahrnehmung der Demokratie durch die Bürger öffentlich machen und damit dazu beitragen, die als Problem wahrgenommen Bereiche zu identifizieren und breit zu thematisieren. Schließlich sind Umfragen geeignet, um die Responsivität der Politik gegenüber den Präferenzen der Bürgerinnen und Bürger zu bewerten. Allerdings ist Responsivität nur bedingt geeignet, um die Qualität einer Demokratie zu messen. ${ }^{52}$

Indikatoren der politischen Kulturforschung können also durchaus zur Bestimmung der Qualität von Demokratie herangezogen werden, allerdings nur unter bestimmten Bedingungen und im Bewusstsein der damit verbundenen Restriktionen. Sie sind somit kein gleichwertiger Weg der Demokratiemessung im Vergleich mit den „quasi-objektiven“ Strategien. Sie können aber sehr wohl in ausgewählten Bereichen als sinnvolle Ergänzung und zur Kontrolle anderer Messungen eingesetzt werden. ${ }^{53}$ Weitaus wichtiger sind sie in der Bestimmung der Stabilität und Konsolidierung einer Demokratie. ${ }^{54}$

\section{Fazit und Perspektiven der Demokratiemessung}

Die Überlegungen zur Bestimmung der Qualität einer Demokratie haben gezeigt, dass es sich um kein einfaches Unterfangen handelt. Darauf verweist gerade die Vielfalt der vorliegenden Messanlagen. Der Einbezug differenzierter qualitativer Ansätze verdeutlicht sowohl die Grenzen der bestehenden Datensätze als auch die Möglichkeiten einer systematischen Analyse demokratischer Systeme. Bei diesen komplexen Verfahren kommt es weniger auf die Indexbildung als auf die Profilanalyse der jeweiligen Demokratie an. Auf diese Weise lassen sich präziser

52 Lauth, H.-J.: Quality Criteria for Democracy. Why Responsiveness is not the Key, in: Erdmann, G./Kneuer, M. (Hg.): Regression of Democracy, Wiesbaden, 2011 (i.E.).

53 Dazu gehört auch der Einsatz von Indikatoren der politischen Kulturforschung für die Messung informeller Institutionen, deren Wirksamkeit sich auf das Verhalten und die Orientierungen der Bürger erstreckt. Hierbei wird von der Annahme ausgegangen, dass sich die Existenz informeller Einrichtungen in den Einstellungen und Kenntnissen der Bürger manifestiert.

54 Konsequenterweise spricht der Report UNDP, a.a.O., bei der Auswertung von Umfragedaten von der Bildung eines Democratic Support Index. 
die Errungenschaften und Schwachstellen der Demokratien feststellen, die neben den formalen Institutionen auch die informellen Strukturen und Praktiken berücksichtigen. Diesbezügliche Untersuchungen zu einem der Mutterländer der Demokratie (Großbritannien) können verdeutlichen, dass auch dort noch deutliche Steigerungspotentiale erkennbar sind. Dies gilt auch für etablierte Demokratien mit regressiven Tendenzen - wie Italien unter Berlusconi. Hier könnte allerdings ein Regierungswechsel bereits den zentralen Kritikpunkt der Medienkonzentration entschärfen. Auch wenn viele theoretische und methodische Kritikpunkte hinsichtlich der einzelnen Messanlagen vorliegen, erlaubt die sorgfältige Verwendung vorliegender Daten, die Qualität der Demokratie einzuschätzen.

Die in diesem Beitrag vorgestellten Messansätze verfolgen erkennbar unterschiedliche Ziele mit divergenten Strategien. So sind der Democratic Audit und IDEA stark an politischen Reformmaßnahmen interessiert, um die Qualität der Demokratie zu steigern. Daher ziehen sie bewusst politische Akteure in den Evaluationsprozess ein und verstärken ihre Öffentlichkeitsarbeit. Während beide Ansätze ein sehr umfassendes Demokratieverständnis ausbreiten, das stark in den gesellschaftlichen Raum reicht, arbeiten Abromeit und Stoiber mit einem sehr schlanken Demokratiebegriff, der sich auf die effektive Partizipation konzentriert. Zudem flexibilisieren sie mit dem Angemessenheitsmaß, das den Beteiligungsbedarf mit dem Partizipationsangebot abgleicht, den allgemeinen Maßstab. Demgegenüber setzt die Demokratiematrix auf eine systematische Erfassung des gesamten demokratischen Raumes und nimmt somit eine Mittelposition hinsichtlich der beiden anderen Forschungseinrichtungen ein. Ebenso wie der Democratic Audit und IDEA betreibt die Demokratiematrix einen erheblichen Forschungsaufwand, um eigene Daten zu erheben. Dieser Aufwand fällt bei Abromeit und Stoiber aufgrund der weitgehenden Abstinenz bei der Erfassung von Akteurshandeln geringer aus, wenngleich die Berechnung der Daten einen Mehraufwand darstellt. Alle drei Forschungswege sind in der Lage, Defizite in der Qualität der Demokratie zu identifizieren, wenngleich die Validität der Messung variiert. Die Demokratiematrix arbeitet zudem mit der Unterscheidung von demokratischen Subtypen und Regimetypen, auf die die anderen Ansätze verzichten.

Die Kontextrelevanz der Demokratiemessung, die im interkulturellen Vergleich von besonderer Bedeutung ist, wird im Unterschied zu den gängigen quantitativen Messanlagen in allen behandelten Ansätzen reflektiert. Allerdings werden andere Lösungen angeboten. So setzen der Democratic Audit und IDEA die 
Stellschraube bereits auf der Ebene der Demokratiedefinition und vor allem der Setzung von Standards an, die den jeweiligen nationalen Bedürfnissen und Vorstellungen entsprechen. Abromeit und Stoiber stellen dem das Kriterium der Angemessenheit entgegen. Demnach ändert sich der institutionelle Maßstab mit Bezug auf das jeweilige partizipatorische Anforderungsprofil. Das Autorenduo formuliert dazu feststehende Lösungen für bestimmte Problemsettings. Lauth schlägt demgegenüber die Beachtung funktionaler Äquivalente vor, sodass die gleiche Funktion durch unterschiedliche institutionelle Arrangements ausgefüllt werden kann. Diese werden im Konzept nicht präzisiert, um kontextsensibel die empirischen Befunde bewerten zu können.

Zwei Aspekte sind am Ende grundsätzlich zu reflektieren. Der eine betrifft nochmals den Demokratiebegriff selbst und der andere die empirischen Veränderungen in den Demokratien. Der Überblick über das gesamte Feld der Demokratiemessung verdeutlichte, dass mit Blick auf den Demokratiebegriff weiterhin kein Konsens besteht, auch wenn sich die Tendenz zu einem mehrdimensionalen Verständnis abzeichnet. Zugleich stellt man fast durchgängig auf den Standard etablierter Demokratien ab und lässt mögliche und notwendige Entfaltungsmöglichkeiten außer Acht. Solche Steigerungspotentiale liegen in der Verbesserung der Rechtsstaatlichkeit (Transparenz und Kontrolle) und in der qualitativen Anhebung der politischen Partizipation, wie sie im Rahmen von deliberativer Demokratie und direkter Demokratie diskutiert wird. ${ }^{55}$ Erste Vorschläge zur Messung deliberativer Demokratie liegen vor und könnten in die vorgestellten Ansätze eingebaut werden. ${ }^{56}$ Auch direktdemokratische Verfahren, die sinnvoll die repräsentative Demokratie ergänzen können, lassen sich in diese Auswertung einbeziehen. Einen brauchbaren Vorschlag zur Bestimmung der Intensität und Qualität direktdemokratischer Prozesse bietet das Ranking-Verfahren, das von dem Fachverband „Mehr Demokratie“ eingesetzt wird. ${ }^{57}$

Zweitens wird deutlich, dass sich die empirischen Referenzbereiche der Demokratie verändern, wobei allerdings noch unklar ist, welche Muster sich letztlich herausbilden werden. Mit der Internationalisierung politischer Räume ist ein

55 Offe, C. (Hg.): Demokratisierung der Demokratie. Diagnosen und Reformvorschläge, Frankfurt/Main, 2003; Schiller, T.: Volksinitiativrechte in Europa - ein vergleichender Überblick, in: Gornig, G.H./Kramer, U./Volkmann, U. (Hg.): Staat - Wirtschaft - Gemeinde, Berlin, 2007, 301-316; Zittel, T./Fuchs, D. (Hg.): Participatory Democracy and Political Participation. Can participatory engineering bring citizens back in?, London/New York, 2007.

56 Vgl. Bächtiger, A./Steiner, J. (Hg.): Acta Politica: Special Issue on Empirical Approaches to Deliberative Democracy, 40/2 \& 40/3 (2005).

57 Mehr Demokratie (Hg.): Ranking. Volksentscheids-Ranking 2010, Berlin 2010. 
Aspekt hervorzuheben, der die Demokratie jedoch unausweichlich betrifft. So ist es fast überraschend, dass dieser Faktor nur bei IDEA eine prominentere Rolle spielt und auch hier nicht durchgängig überzeugt. Eigentlich müsste bei allen Evaluierungs- und Messverfahren, die sich auf EU-Mitgliedstaaten richten, auch die demokratische Qualität der EU berücksichtigt werden, da von einem abgeschlossenen nationalen politischen System nicht mehr gesprochen werden kann. ${ }^{58}$ Im Umkehrschluss beeinflusst die Demokratiequalität der EU auch die der Einzelstaaten. ${ }^{59}$ Da die Qualität der Demokratie in der EU tendenziell eher skeptisch betrachtet wird, müssten folglich alle Mitgliedstaaten abgewertet werden. Allerdings wird die EU auch als System sui generis betrachtet und bedarf daher auch spezifischer Maßstäbe. Da es sich mit der EU und den Mitgliedstaaten um ein integrales System handelt, in dem funktionale Kompensationen möglich sind, sollten beide - EU und die Mitgliedstaaten - nicht separat erfasst werden, sondern bedürften eines integralen Messkonzepts, das bislang noch nicht vorliegt. Um eine angemessene Einschätzung der Demokratie in den EUMitgliedsländern zu erzielen, sollte diese Perspektive stärker als bisher berücksichtigt werden. Doch auch jenseits der EU besteht Bedarf, die internationale Ebene stärker hinsichtlich ihrer Bedeutung für die Qualität der Demokratie zu erfassen. Wenn bei wachsender Sensibilität und Vulnerabilität von Interdependenzstrukturen externe Faktoren eine zunehmende Bedeutung für nationale Entscheidungsprozesse gewinnen - wie im Zuge der Bankenkrise deutlich wurde dann sind auch hier die Konsequenzen für die Demokratiemessung zu prüfen. Schließlich wäre zu diskutieren, inwieweit das Verhalten von Staaten jenseits ihrer nationalen Grenzen von Relevanz für die Demokratiebewertung ist. Die Reaktionen der USA in ihrem Kampf gegen den Terrorismus werfen solche Fragen auf.

Allein die Bemerkungen zu den letzten beiden Punkten unterstreichen den konzeptionellen Forschungsbedarf auf dem Terrain der Demokratiemessung. Dabei sollten deren Grenzen, aber auch das bereits vorhandene Leistungspotential beachtet werden. So wäre es zu begrüßen, wenn in der weiteren Forschung die Anwendung der Konzepte auf eine größere empirische Basis gestellt werden könnte. Die präsentierten Ansätze bieten vielfältige Möglichkeiten der Umsetzung.

58 Sturm, R./Pehle, H.: Das neue deutsche Regierungssystem, Stuttgart, 2005.

59 Dies wird von Democratic Audit konzeptionell durchaus erfasst, jedoch nur begrenzt empirisch umgesetzt. 